\title{
How to detect supermassive binary black holes at parsec scales
}

\author{
Xiang Liu \\ Xinjiang Astronomical Observatory of CAS, 150 Science 1-Street, Urumqi 830011, China \\ email: liux@xao.ac.cn
}

\begin{abstract}
It is difficult to find or identifying the binary black holes in parsec scales, since the dual AGN may be merged quickly. It is required to explore more possibilities to identifying binary black holes in parsec scales, we give some discussions, especially with the VLBI methods.
\end{abstract}

Keywords. black hole physics: binary - galaxies: jets - quasars: general - radio continuum

It is possible that supermassive binary black holes live in active galaxies through merging process. For the huge gravitational potential of massive black hole and the angular momentum losses via gas accreting and emission, the binary massive black holes will be gradually merged to form a larger black hole. It is not clear that the time scale of the evolution from far separation of two massive black holes to kpc scale separation, and the time scale from the $\mathrm{kpc}$ scale to pc scale separation. Statistical studies seem to suggest that the detection rate of pc-scale binary black holes $(\mathrm{BBH})$ is much less than that of the kpc-scale dual AGN (Smith et al. 2010), this implies the inspiral of dual black holes may be faster in the pc scale than in the kpc scale (Blecha et al. 2013). It is difficult to find or identifying the binary black holes in the pc scale, since the dual AGN would be to merge quickly. It is required to explore more possibilities to identifying binary black holes in the pc-scales, we give some discussions in the following.

High spectral resolution to reveal the double-peaked broad lines. When supermassive binary black holes with similar masses have their own accretion disks, the two set of similar emission lines could be detectable. The double-peaked emission line AGN found by Wang et al. (2009), some of them were identified to show dual AGN in the kpc scale. When the dual AGN evolve into pc-scale, their narrow-line regions can be jointed, but the two broad-line regions may be not jointed yet, and their broad emission-lines could be doublepeaked and identifiable. One needs higher spectral resolution and sensitivity to reveal the double-peaked broad lines. Furthermore, it may also be possible to associate the pc-scale $\mathrm{BBH}$ candidate with the line-of-sight radial velocity shifts of the broad lines if attributing the shift to the orbital motion of BBH, from multi-epoch spectral monitoring (Liu et al. 2014).

VLBI detection of double twin-jets from binary black holes at pc-scale. Very long baseline interferometry (VLBI) at radio can resolve the radio loud AGN at pc scales. Tens of thousands AGN have been imaged with the high resolution, but the binary black holes in AGN with the VLBI were not investigated systematically. Assuming radio loud AGN fraction is $10 \%$, the fraction for both the dual AGN are radio loud will be $1 \%$, this means for the $1 \%$ of dual AGN that we should be able to detect the double twin-jets from their binary black holes. Only one AGN has been identified with such double twinjets/cores, PKS 0402+379 (Rodriguez et al. 2006), with two flat spectrum radio cores of $\sim 7$ pc apart. Burke-Spolaor (2011) searched for flat spectrum radio cores from the geodetic VLBI database, found again only the PKS 0402+379. We have searched for binary black holes from the astrophysical VLBI databases, found $5 \mathrm{BBH}$ candidates from 


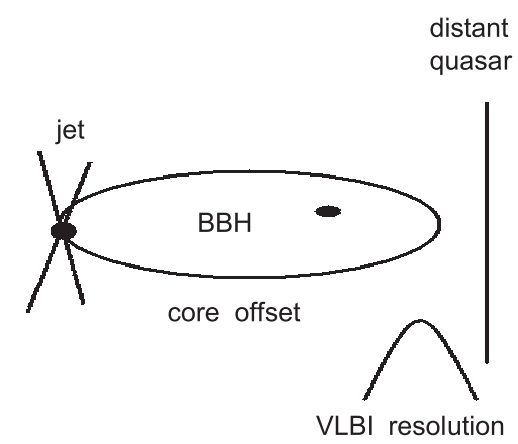

Figure 1. The schematic diagram of the phase-reference VLBI method to detect the core-offset of the radio loud core in a binary black hole system.

$\sim 2000$ sources. Our strategy of searching for BBH candidates is looking for not only flat spectrum double cores, but also double twin-jets pair or their variant complex (Liu 2014). However, most of the VLBI-imaged jets we investigated are limited in spatial resolution at centimeter bands, further investigations to confirm the $\mathrm{BBH}$ candidates are ongoing through high and multi-frequency VLBI observations.

VLBI core-offset to be determined as due to the BBH orbital motion. For binary black hole AGN, with radio loud probability $10 \%$ of each hole, it is likely that only one $\mathrm{BH}$ is jetting and the other $\mathrm{BH}$ is radio quiet in most of dual AGN. In this case, one cannot detect twin jets from both black holes. The BL Lac object OJ287, for instance, is believed to host a BBH system for its nearly 12 years periodic optical/radio outbursts that attributing to the orbital motion of BBH. The VLBI image of OJ287 shows only one-sided jet, exhibiting large position-angle changes of inner-jets at high frequencies (Liu et al. 2012) and with the core-shifts between different frequencies in VLBI images as well. The core shifts are mainly caused by the opacity effect of inner jets. The position-angle changes of inner jets, however, may be caused by the precession or orbital motion of the $\mathrm{BBH}$ system. Therefore, with multi-epoch VLBI observations at same frequency, one would be able to detect the orbital motion of the radio loud $\mathrm{BH}$ in the $\mathrm{BBH}$ system if the other is radio quiet, the so-called 'core offset', with the phase-reference VLBI technique (see Fig. 1), as referenced to a distant high-redshift quasar (which has no detectable coreoffset). This needs a long time to measure the core offset caused by the pc-scale orbital motion of $\mathrm{BBH}$, but it is plausible to detect for the binary black holes at low redshift.

\section{References}

Blecha, L., Loeb, A., \& Narayan, R. 2013, MNRAS, 429, 2594

Burke-Spolaor, S. 2011, MNRAS, 410, 2113

Liu, X., Shen, Y., Bian, F. Y., et al. 2014, ApJ, 789, 140

Liu, X. 2014, J. Astrophys. Astr., Vol.35, No.3, 1

Liu, X., Mi, L.-G., Liu, B.-R., \& Li, Q.-W. 2012, ApछSS, 342, 465

Rodriguez, C., Taylor, G. B., Zavala, R. T., et al. 2006, ApJ, 646, 49

Smith, K. L., et al. 2010, ApJ, 716, 866

Wang, J.-M., et al. 2009, ApJ (Letters), 705, L76 